Agric. Biol. Chem., 42 (4), $711 \sim 716,1978$

\title{
The Mechanism of Synergism between Tocopherols and Trimethylamine Oxide in the Inhibition of the Autoxidation of Methyl Linoleate
}

\author{
Yukihiro IshIKawa, Etsuji YuKI, ${ }^{*}$ Hiromichi Kato** \\ and Masao Fujmaki** \\ Faculty of Education, Tottori University, Tottori 680 \\ *Hiroshima Food Research Institute, Hiroshima 730 \\ **Department of Agricultural Chemistry, The University of Tokyo, Tokyo 113
}

Received July 13, 1977

\begin{abstract}
The synergistic effect of trimethylamine oxide (TMAO) on the antioxidant activity of $\gamma$-tocopherol $(\gamma$-Toc) to inhibit the autoxidation of methyl linoleate was investigated.

Formation of the reducing dimers of $\gamma$-Toc was found to be closely related to the synergism of TMAO with $\gamma$-Toc. $\gamma$-Toc diphenyl ether dimer and two $\gamma$-Toc biphenyl dimers were identified, and the latters were considered to be atropisomers with each other.

From the reaction of the hydroperoxide ( $\mathrm{LOOH}$ ) of methyl linoleate with TMAO, TMAO was found to act as a peroxide decomposer. TMAO produced methyl keto-octadecadienoate, which was formed only in the presence of $r$-Toc and TMAO, from LOOH, and markedly decreased the POV of LOOH.

A possible mechanism of synergism between Toc and TMAO in the inhibition of the autoxidation of methyl linoleate was proposed.
\end{abstract}

Tocopherol (Toc) is widely present in vegetable oils and has been expected to replace some synthetic antioxidants such as BHA and BHT. Although the antioxidative activity of Toc is inferior to that of some synthetic antioxidants, it may be expected to have a marked antioxidative activity in a combination with proper synergists. Substances such as amino acid, ${ }^{, \sim 3}$ ) melanoidin, ${ }^{4 \prime}$ flavonoids, ${ }^{51}$ amines, ${ }^{6)}$ phospholipid, ${ }^{7 \sim 8 i}$ ascorbic acid, ${ }^{9 \sim 10}$ citric acid, ${ }^{10 \sim 12)}$ BHA, ${ }^{13}$ trimethylamine oxide (TMAO), etc. have been known to be synergists of Toc. We have made much effort to clarify the mechanism of antioxidative synergism between Toc and TMAO, which exists in fish.

In a previous paper, ${ }^{15)}$ synergism between $\delta$ Toc and TMAO in the inhibition of the autoxidation of methyl linoleate was found to be closely related to the formation of the reducing dimers of $\delta$-Toc. Furthermore, methyl linoleate containing $\delta$-Toc and TMAO characteristically formed methyl keto-octadecadienoate (abbreviated as keto acid), and its peroxide value (POV) did not increase much.

Kamiya $^{16,17)}$ described synergism between a free radical acceptor and a peroxide decomposer which occur in the following way. A free radical acceptor (e.g., Toc or its dimers) acts on the peroxy radical of oil to form hydroperoxide. The hydroperoxide reacts with a peroxide decomposer to produce inactive compounds, which do not act as initiators of a radical chain reaction any longer.

Yamaguchi et al. reported that melanoidin acted as a synergist when coupled with Toc and decomposed peroxides in a hydrated system. $^{4,18)}$ However, the synergistic mechanism has never been clarified.

The present paper deals with the reaction of $\alpha$ - and $\gamma$-Toc with TMAO during the autoxidation of methyl linoleate compared with that of $\delta$-Toc. ${ }^{15)}$ The roles of TMAO in the formation of the keto acid from methyl linoleate and in decreasing POV were also investigated, and a possible synergistic mechanism between Toc and TMAO is proposed.

\section{MATERIALS AND METHODS}

Toc. $\quad d l-\alpha-\operatorname{Toc}(100 \%)$ and $d-\gamma-\operatorname{Toc}(98 \%)$ were 
kindly provided by Eisai Co. and used without further purification.

Methyl linoleate. Methyl linoleate was the same as used in the previous paper. ${ }^{13)}$

Methyl linoleate hydroperoxide. The hydroperoxide (LOOH) was prepared from autoxidized methyl linoleate according to the method of Matsushita et al., ${ }^{19}$ ) and used after purification by TLC.

TMAO. TMAO (Tokyo Kasei Co.) of analytical grade was purchased.

Isolation of reaction products. Reaction products of $\alpha$ - and $\gamma$-Toc and methyl linoleate were isolated and their amounts were determined by weighing after purification according to the method described in the previous paper..$^{15)}$

Measurement of POV. POV was iodometrically determined.

Evaluation of induction peroid. The experimental procedures were the same as those in the previous paper. ${ }^{15}$

Decomposition of $\mathrm{LOOH}$. A mixture of $\mathrm{LOOH}$ and TMAO or amine was kept under nitrogen in the dark at $50^{\circ} \mathrm{C}$. The degree of the decomposition of LOOH was followed by measuring POV. After treatment, the reaction mixture was subjected to TLC with a solvent system, $n$-hexane ethyl ether $(8: 2, v / v)$.

$T L C$. Thin-layer plates (thickness, $0.5 \mathrm{~mm}$ ) were prepared with Kieselgel $\mathrm{HF}_{254}$ (Merck), and the chromatograms were examined under ultraviolet light and with reagents such as 2,4-dinitrophenylhydrazine and potassium iodide.

\section{RESULTS}

1. Effect of TMAO on the antioxidant activity of $\alpha$ - and $\gamma-T o c$

1) Degradation of $\gamma$-Toc. Changes in the amount of $\gamma$-Toc during the autoxidation of methyl linoleate with and without TMAO were followed by $\mathrm{GLC}^{15}{ }^{15}$ and the results are shown in Fig. 1.

$\gamma$-Toc in the reaction mixtures treated with and without TMAO seemed to disappear within almost the same time. Methyl linoleate containing $\gamma$-Toc and TMAO needed a longer induction period of 26 days to initiate propagation even after a drastic decrease in the amount of $\gamma$-Toc compared with that of 9 days

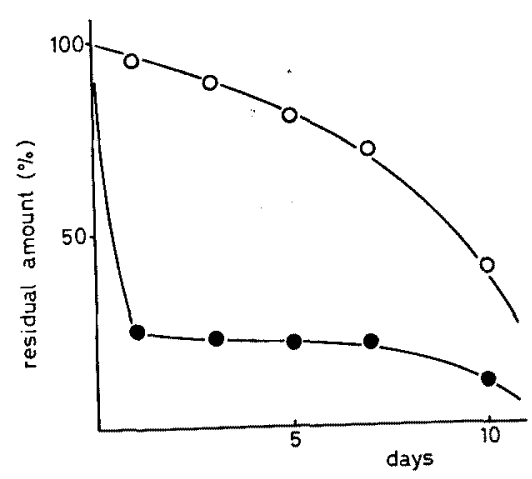

FIG. 1. Degradation of $r$-Tocopherol during the Autoxidation of Methyl Linoleate.

Methyl linoleate containing $0.1 \%$ of $\gamma$-Toc and $0.05 \%$ of TMAO was kept in the dark at $60^{\circ} \mathrm{C}$. O, without TMAO; $\bullet$, with TMAO.

in the absence of TMAO. The results suggest that synergism between $\gamma$-Toc and TMAO mainly depends on the interaction of the reducing dimers derived from $\gamma$-Toc with TMAO in the autoxidizing oil. ${ }^{20}$

2) Changes in the amounts of $\gamma$-Toc and its dimers. Three dimers of $\gamma$-Toc were obtained when methyl linoleate containing $4 \%$ of $\gamma$-Toc and $2 \%$ of TMAO was autoxidized in the dark at $50^{\circ} \mathrm{C}$, and they were identified to be $5-(\gamma-$ tocopheryl-oxy)- $\gamma$-tocopherol ( $\gamma$-Toc diphenyl ether dimer) and two 5-( $\gamma$-tocopheryl)- $\gamma$ tocopherol ( $\gamma$-Toc biphenyl dimers) which are existing as atropisomers with each other as described in a previous paper, ${ }^{20}$ Changes in the amounts of $\gamma$-Toc and its dimers were followed in the presence or absence of TMAO, and the results are shown in Fig. 2.

The induction period for the autoxidation of methyl linoleate was about 30 days in the absence of TMAO, and $\gamma$-Toc and its dimers disappeared in about 35 days, while the period in the presence of TMAO was more than 150 days and remarkable synergism was observed. The change in POV proceeded almost in the same manner as in the case of $\delta$-Toc reported previously. ${ }^{15}$ )

Figure 3 shows the relationship between the amount of the keto acid formed and the consumption of $\gamma$-Toc and its dimers. The amounts of keto acid formed only in the pre- 

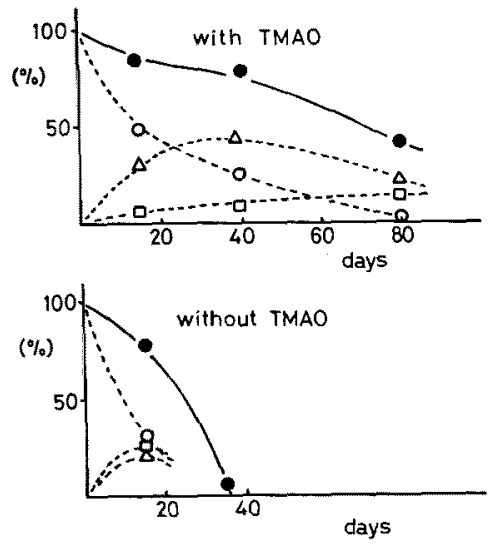

FIG. 2. Changes in the Amounts of $\gamma$-Tocopherol and Its Dimers during the Autoxidation of Methyl Linoleate with and without TMAO.

Autoxidized methyl linoleate of a commercial product was purified by passing through a silica gel column to remove peroxides. The purified methyl linoleate containing $4 \%$ of $\gamma-\mathrm{Toc}$ and $2 \%$ of TMAO was autoxidized in the dark at $50^{\circ} \mathrm{C}$. $\quad$, total amounts of $\gamma$-Toc and its dimers; $0, \gamma$-Toc; $\gamma, \Delta$-Toc biphenyl dimers; $\square, \gamma$-Toc diphenyl ether dimer.

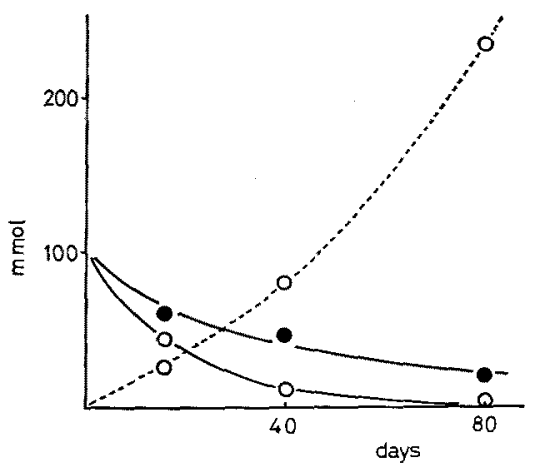

FIG, 3. Relationship between the Formation of Methyl Keto-octadecadienoate and the Consumption of $\gamma$-Tocopherol and Its Dimers during the Autoxidation of Methyl Linoleate Containing TMAO.

The incubation conditions were the same as those described in Fig. 2 . O-O, $\gamma$-Toc; $-0, r$-Toc and its dimers; $O-\circ$, methyl keto-octadecadienoate.

sence of TMAO were $0.8,2.4$, and $7.3 \%$ of the initial weight of methyl linoleate at 16,40 , and 80 days reaction, respectively.

3) Changes in the amounts of $\alpha-T o c$ and keto acid. Figure 4 shows changes in the amounts of $\alpha$-Toc and keto acid during the autoxidation of methyl linoleate at $50^{\circ} \mathrm{C}$.

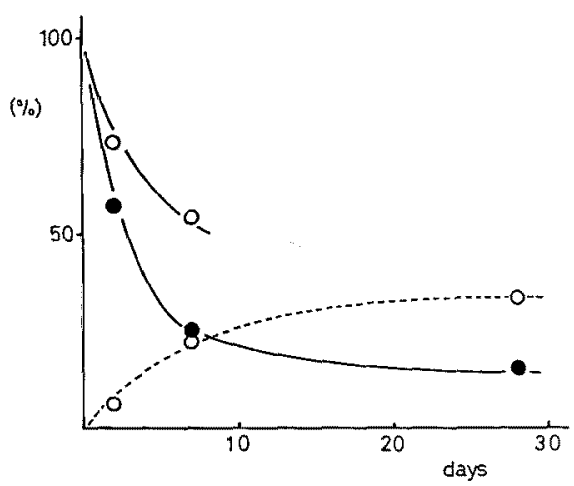

FIG. 4. Degradation of $\alpha$-Tocopherol and Formation of Methyl Keto-octadecadienoate during the Autoxidation of Methyl Linoleate.

Methyl linoleate containing $4 \%$ of $\alpha$-Toc and $2 \%$ of TMAO was autoxidized in the dark at $50^{\circ} \mathrm{C}$.

- and O-O, $\alpha$-Toc with and without TMAO, respectively; $\mathrm{O}--\mathrm{O}$, methyl keto-octadecadienoate.

$\alpha$-Tocopheryl ethane, a dimer formed from the reaction of $\alpha$-Toc with TMAO as described in a previous paper ${ }^{20}$, was not observed. NMR analysis showed that a complex between $\alpha$-Toc and methyl linoleate was present in the reaction products, but its structure could not be determined.

A noteworthy feature in the presence of TMAO was the formation of a large amount of keto acid, and about one-third of methyl linoleate changed into keto acid at 28 days after the initiation of reaction.

\section{Reaction of $\mathrm{LOOH}$ with TMAO}

In order to clarify the causes of the inhibition of the increase in POV and the pathway for keto acid, investigated were reactions of TMAO with $\mathrm{LOOH}$ which is the first oxidation product in the autoxidation of methyl linoleate and is produced by reaction between an antioxidant and methyl linoleate peroxy radical. $^{21 \sim 23)}$

1) Decomposition of LOOH with TMAO. The results are shown in Fig. 5. As LOOH is unstable, it decomposed under nitrogen at $50^{\circ} \mathrm{C}$ and the decrease in POV was observed. The drastic decrease in $\mathrm{POV}$ in the presence of TMAO indicates that $\mathrm{LOOH}$ was rapidly decomposed and TMAO acted as a peroxide 


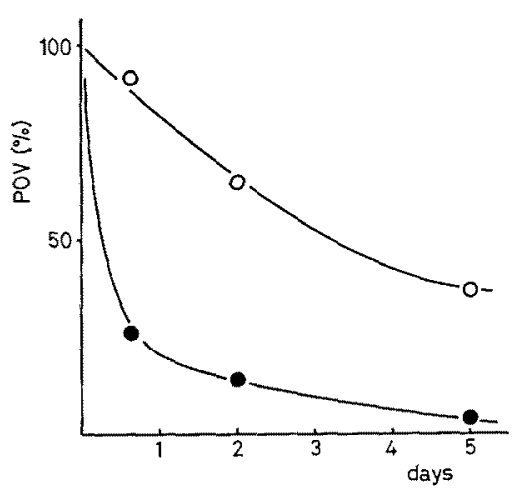

FIG. 5. Decomposition of Methyl Linoleate Hydroperoxide (LOOH) with TMAO.

LOOH $(1 \mathrm{~g})$ with and without TMAO $(100 \mathrm{mg})$ was kept under nitrogen in the dark at $50^{\circ} \mathrm{C}$.

Initial POV of $\mathrm{LOOH}$ was 5240 .

$O$, without TMAO;, with TMAO.

decomposer. ${ }^{16,17)}$

2) TLC of $\mathrm{LOOH}$ treated with TMAO. As shown in Fig. 6, a characteristic spot was observed at $R f 0.4$ corresponding to keto acid, and its NMR spectrum coincided with that of an authentic sample. ${ }^{15)}$ However, no spot at Rf 0.4 was observed in $\mathrm{LOOH}$ without TMAO.

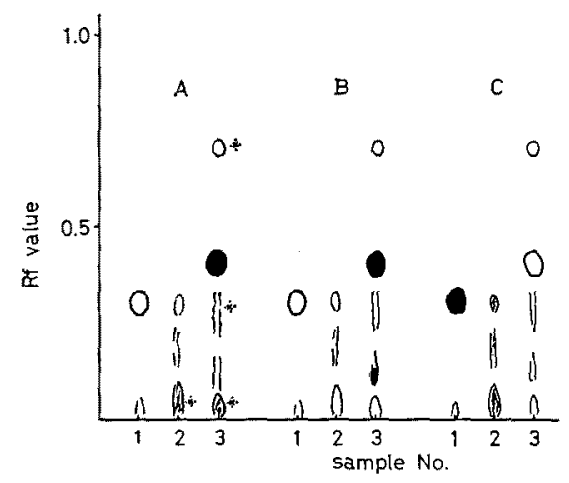

FiG. 6. Thin-layer Chromatograms of Methyl Linoleate Hydroperoxide Treated in the Presence or Absence of TMAO.

TLC: Kieselgel $\mathrm{HF}_{254}$, $n$-hexane-ethyl ether $(8: 2)$.

Detection: A, UV light at $254 \mathrm{~nm}$; B, 2,4-DNPH; $C$, potassium iodide.

Sample: 1, original LOOH, 2, LOOH incubated without TMAO; 3, LOOH incubated with TMAO.

Samples 2 and 3 were the same as those in Fig. 5 (incubated for 5 days).
3) Decomposition of $\mathrm{LOOH}$ with amines. As shown in Table I, tri- $n$-octylamine and stearylamine, showing synergism with Toc, ${ }^{24}$ acted as a potent decomposer, but diphenylamine, known as a peroxide decomposer, ${ }^{25}$ rather inhibited the decomposition of $\mathrm{LOOH}$. On TLC (Fig. 7) the formation of much keto acid was observed in $\mathrm{LOOH}$ treated with TMAO followed by tri-n-octylamine.

Table I. Decomposition of Methyl Linoleate Hydroperoxide with AMINES

LOOH $(45 \mathrm{mg})$ was incubated with amine $(1 \mathrm{M})$ under nitrogen in the dark at $50^{\circ} \mathrm{C}$, and POV $(\%)$ after 3 days of incubation was expressed as the percentage of the initial POV of LOOH (100).

\begin{tabular}{lc} 
& POV $(\%)$ \\
\hline LOOH (original) $^{a}$ & 100 \\
TMAO & 4.3 \\
Tri-n-octylamine & 0 \\
Stearylamine & 0 \\
Diphenylamine & 49.7 \\
LOOH (incubated) & 37.9 \\
\hline$a$
\end{tabular}

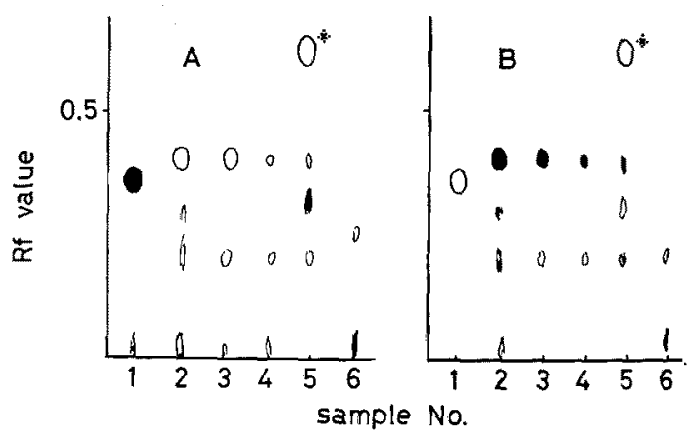

FIG. 7. Thin-layer Chromatograms of Methyl Linoleate Hydroperoxide Treated with Amines.

TLC: the same as in Fig. 6.

Detection: A, potassium iodide; B, 2,4-DNPH.

Sample: 1 , original LOOH; 2 , TMAO; 3 , tri-n-octyl amine; 4 , stearylamine; 5 , diphenylamine; 6 , LOOH incubated.

Samples were the same as in Table I.

* : diphenylamine.

\section{DISCUSSION}

The effect of TMAO on the antioxidant activity of $\alpha$ - and $\gamma$-Toc in methyl linoleate was investigated. 
$\delta$-Toc was found to have remarkable synergism with TMAO and to form its reducing dimers and keto acid from methyl linoleate, as found similarly in the case of $\delta$-Toc reported previously. ${ }^{15 /}$ On the contrary, synergism between $\alpha$-Toc and TMAO could not be expected because TMAO accelerated the autoxidation of methyl linoleate in an early stage of the reaction. The POV of the oil with TMAO, therefore, increased more rapidly than without TMAO within 1 day, but this relation was reversed after 2 days.

Although synergism between $\gamma$-Toc and TMAO was observed under the conditions described in Figs. 1 and 2, large differences in the rate of the decrease of $\gamma$-Toc in both cases might be related to the concentration of Toc, $^{26,27)}$ temperature ${ }^{27,28)}$ and the degree of autoxidation of the substrate. In the last case, it was observed that $\gamma$-Toc decreased to near zero after 6 days when commercial methyl linoleate (POV, ca. 15) was autoxidized under the conditions described in Fig. 2.

An initial concentration of $4 \%$ of $\gamma$-Toc is approximately equivalent to $100 \mathrm{mmol} / \mathrm{kg}$. When $\gamma$-Toc $(\mathrm{AH})$ reacts with the peroxy radical (LOO·), a hydrogen atom of an $\mathrm{OH}$ group of the $\mathrm{AH}$ is abstracted and a A radical is given. It readily combines each other and forms a reducing dimer (AAH). In this case, the total effective amounts of $\mathrm{AH}$ and $\mathrm{AAH}$ should be equivalent to about $150 \mathrm{mmol} / \mathrm{kg}$. If $1 \mathrm{~mol}$ of methyl linoleate is autoxidized, it forms initially the alkyl radical $(\mathrm{L} \cdot)$, which reacts rapidly with oxygen to give the LOO. radical, and consumes $1 \mathrm{~mol}$ of $\gamma$-Toc or its dimers to form $1 \mathrm{~mol}$ of $\mathrm{LOOH}$, which decomposes to produce $1 \mathrm{~mol}$ of keto acid. Therefore, in the experiment in Fig. 3 , if $\mathrm{AH}$ and AAH are completely consumed, $150 \mathrm{mmol}$ of keto acid should be formed. However, as shown in Fig. 3, about $20 \mathrm{mmol} / \mathrm{kg}$ of the dimers remained in the reaction mixture, whereas about $230 \mathrm{mmol} / \mathrm{kg}$ of keto acid was formed after the reaction for 80 days. Furthermore, the amount of $\gamma$-Toc decreased gradually, whereas its dimers was found to be kept at a constant concentration. These results suggest that the autoxidation of methyl linoleate should be inhibited owing to synergism between the reducing dimers and TMAO after $\gamma$-Toc disappeared, and that most reducing dimers in the presence of TMAO may be regenerated by repeated reduction.

From the reaction of LOOH with TMAO, it was found that the inhibition of the increase in POV and the formation of keto acid mainly depends on the action of TMAO as a peroxide decomposer. A similar mechanism is also considered in the case of synergism between $\delta$-Toc and TMAO, described in the previous paper. ${ }^{15)}$

Although various pathways have been reported for the formation of keto acid by reacting $\mathrm{LOOH}$ with some substances, ${ }^{25,29 \sim 33}$; it is considered that TMAO produces keto acid from $\mathrm{LOOH}$ in the following way:

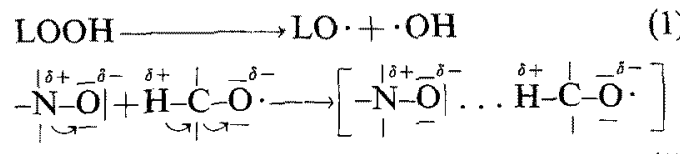

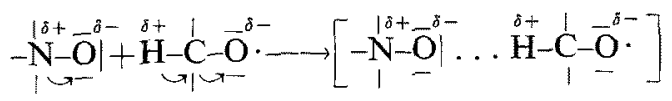

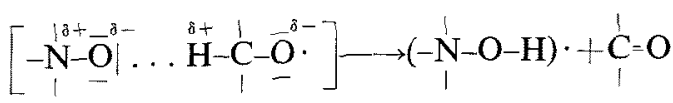

$$
\mathrm{LOOH}+\mathrm{TMAO} \rightarrow(\mathrm{TMAO}-\mathrm{H}) \cdot+\mathrm{L}=\mathrm{O}+\cdot \mathrm{OH}
$$

An active intermediate, $(\mathrm{TMAO}-\mathrm{H}) \cdot$, which is assumed to be formed when TMAO abstracts $\mathrm{H}^{\cdot}$ from the alkoxy radical ( $\mathrm{LO} \cdot$ ) to give keto acid $(\mathrm{L}=\mathrm{O})$, may react easily with an oxidation product of Toc dimer $\left(\mathrm{AA}^{\cdot}\right)$ to regenerate an initial reducing dimer (AAH) which repeatedly serves as an antioxidant. Although a remarkable synergistic effect could be obtained by combining an antioxidant with a peroxide decomposer, ${ }^{16,17,33)}$ its mechanism has never been clarified in the case of edible oils and fats. Phenol, ${ }^{17}$ ) amine, ${ }^{33}$ melanoidin, ${ }^{18}$, etc. have been reported as peroxide decomposers, and they are reducing matters. However, TMAO differs from them in the fact that it acts as a potent prooxidant under some conditions. ${ }^{14)}$

From the results, a possible mechanism of synergism between Toc (mainly $\gamma$ - and $\delta$-Toc) 


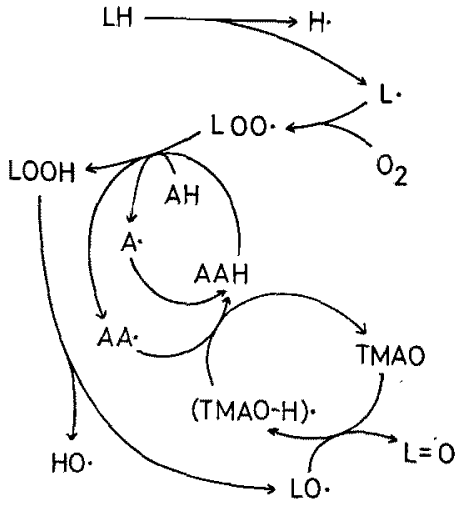

FIG. 8. A Possible Mechanism of Synergism between Tocopherols and TMAO.

and TMAO is likely to be concluded as follows (Fig. 8). Synergism between Toc and TMAO is mainly due to that between the reducing dimers of Toc (AAH) and TMAO. AAH is consumed by offering $\mathrm{H}$ - to LOO radical to give $A A$ radical and $L O O H$. TMAO then reacts with $L O O H$ to produce keto acid and an active intermediate, (TMAO-H) , which offers $\mathrm{H} \cdot$ to $\mathrm{AA} \cdot$ radical, thus $\mathrm{AAH}$ is regenerated.

Acknowledgement. The authors are indebted to Eisai Co. for tocopherol samples.

\section{REFERENCES}

1) J. Janicki and M. Gogolewski, Przemysl Spozywczy, 12, 417 (1958) [C. A., 53, 15602d (1959)].

2) Y. Watanabe and Y. Ayano, J. Jap. Soc. Food Nutr., 25, 219 (1972).

3) E. Yuki, Y. Ishikawa and T. Yoshiwa, Yukagaku, 23, 497, 714 (1974).

4) N. Yamaguchi and M. Fujimaki, J, JPN. Soc. Food Sci. Technol, 21, 13 (1974).

5) N. Yamaguchi, ibid., 22, 270 (1975).

6) H. S. Olcott, ibid., 11, 544 (1964).

7) L. M. Smith, E. N. Frankel, W. Haab and E. L. Jack, J. Dairy Sci., 41, 472 (1958).

8) C. Kanno, K. Yamauchi and T. Tsugo, Agric. Biol. Chem., 34, 1652 (1970).
9) G. Pongracz, Internat. J. Vit. Nutr. Res., 43, 517 (1973).

10) A. L. Tappel, W. D. Brown, H. Zalkin and V. P. Maier, J. Am. Oil Chem. Soc, 38, 5 (1961).

11 E. N. Frankel, P. M. Cooney, H. A. Moser, J. C. Cowan and C. D. Evans, Fette, Seifen Anstrichm., 61, 1036 (1959).

12) S. Masuyama, Yukagaku, 14, 692 (1965).

13) L. Dugan, J. Am. Oil Chem. Soc., 33, 527 (1957).

14) E. Yuki, Y. Ishikawa, I. Yamaoka and T. Yoshiwa, J. JPN. Soc. Food Sci. Technol., 20, 411 (1973).

15) Y. Ishikawa, E. Yuki, H. Kato and M. Fujimaki, Agric. Biol. Chem., 42, 703 (1978).

16) Y. Kamiya, Yukagaku, 14, 672 (1965).

17) Y. Kamiya, "Yuki Sanka Hanno," Gihodo Pub. Co., Tokyo, 1973, pp. 85, 338.

18) N. Yamaguchi and Y. Okada, J. JPN. Soc. Food Sci. Technol., 15, 187 (1968).

19) S. Matsushita, M. Kobayashi and Y. Nitta, Agric. Biol. Chem, 34, 817 (1970).

20) Y. Ishikawa and E. Yuki, ibid., 39, 851 (1975).

21) N. Uri, "Autoxidation and Antioxidants," Vol. 1, ed. by W. O. Lundberg, Interscience Pub., 1961, pp. $134 \sim 6$.

22) W. O. Lundberg, Symposium on Foods, Lipids and Their Oxidation, ed. by H. W. Schultz et al, Avi Pub. Co., 1962, pp. 36 42.

23) J. Pokorny, Can. Inst. Food Technol. J., 4, 68 (1971).

24) Y. Ishikawa, E. Yuki, H. Kato and M. Fujimaki, Yukagaku, 26, 765 (1977).

25) L. Reich and S. S. Stivala, "Autoxidation of Hydrocarbons and Polyolefins, Kinetics and Mechanisms," translated by K. Matsuzaki and Z. Osawa, Maruzen Pub. Co., Tokyo, 1972, p. 152.

26) C. Kanno, M. Hayashi, K. Yamauchi and T. Tsugo, Agric. Biol. Chem., 34, 878 (1970).

27) L.T. Kováts und E. Berndorfer-Kraszner, Nahrung, 12, 407 (1968).

28) Y. Ishikawa and E. Yuki, Yukagaku, 23, 710 (1974).

29) Y. Ogata, "Yuki Kasankabutsu no Kagaku," Nankodo Pub. Co., Tokyo, 1971, pp. 6, 27.

30) M. Hamberg, Lipids, 10, 87 (1975).

31) G. J. Garssen, J. F. G. Vliegenthart and J. Boldingh, Biochem. J., 122, 327 (1971).

32) G. Streckert and H. J. Stan, Lipids, 10, 847 (1975).

33) G. Scott, "Atmospheric Oxidation and Antioxidants," Elsevier Pub. Co., 1965, pp. 188 203. 